\title{
ON THE NONWANDERING SETS OF DIFFEOMORPHISMS OF SURFACES
}

\author{
TOKIHIKO KOIKE
}

\section{§1. Introduction}

Let $M$ be a compact manifold without boundary. Let $f: M \rightarrow M$ be a $C^{1}$ diffeomorphism. Then the nonwandering set $\Omega(f)$ is defined to be the closed invariant set consisting of $x \in M$ such that for any neighborhood $U$ of $x$, there exists an integer $n \neq 0$ satisfying $f^{n}(U) \cap U \neq \phi$. In particular, the set $\operatorname{Per}(f)$ of all periodic points is included in $\Omega(f)$.

Generally, in the study of the orbit structure of diffeomorphisms their nonwandering sets play an essential role. Several results relating to the non-wandering sets established in these ten years or so have developed a new aspect of dynamics - the study of the orbit structure of dynamical systems. In his survey [8], Smale set up a concept called Axiom $A$, i.e. (a) $\Omega(f)=$ $\overline{\operatorname{Per}(f)}$, (b) $T f$ has a hyperbolic structure over $\Omega(f)$, i.e. there exists a $T f$ invariant continuous splitting $E^{s} \oplus E^{u}$ of $T M \mid \Omega(f)$-the restriction of the tangent bundle $T M$ to $\Omega(f)$-such that for some constants $C>0,0<\lambda<1$,

$$
\begin{array}{ll}
\left\|T f^{n}(v)\right\| \leq C \lambda^{n}\|v\|, & \forall v \in E^{s}, \forall n>0, \\
\left\|T f^{-n}(v)\right\| \leq C \lambda^{n}\|v\|, & \forall v \in E^{u}, \forall n>0 .
\end{array}
$$

After that, many important results were obtained in this direction.

On the other hand, Pugh [7] proved a very important theorem about the nonwandering sets. To state it, we shall explain the concept of genericity. Let $\operatorname{Diff}^{1}(M)$ be the set of all $C^{1}$ diffeomorphisms endowed with the $C^{1}$ topology. Then a property of diffeomorphisms is called generic if the diffeomorphisms having it form a residual subset of $\operatorname{Diff}^{1}(M)$.

Pugh's Density Theorem. The property $\Omega(f)=\overline{\operatorname{Per}(f)}$ is generic in $\operatorname{Diff}^{1}(M)$.

Received July 21, 1978.

Revised November 24, 1978. 
In this paper we shall study the nonwandering sets of diffeomorphisms of surfaces from the viewpoint of genericity. Our results are as follows: Let $M^{2}$ be a compact connected surface without boundary.

THEOREM 1. The property that int $\Omega(f),=\phi$, or $f$ is an Anosov diffeomorphism is generic in $\operatorname{Diff}^{1}\left(M^{2}\right)$.

Remark. For a topological space $X$, the closure and the interior of $A \subset X$ are denoted by $\bar{A}$ and $\operatorname{int} A$ respectively.

A diffeomorphism $f: M \rightarrow M$ is called Anosov if $T f$ has a hyperbolic structure over $M$. For surfaces except a torus, there is no Anosov diffeomorphisms ([9], p. 90). So, in this case Theorem 1 is written as follows:

TheOREM $1^{\prime}$. The property int $\Omega(f)=\phi$ is generic in $\operatorname{Diff}^{1}\left(M^{2}\right)$ if $M^{2}$ is not a torus.

A diffeomorphism $f$ is said to be topologically $\Omega$-stable if $\Omega(f)$ is homeomorphic to $\Omega(g)$ for all $g C^{1}$ near $f$. We have the following from Theorem 1.

Corollary. If $f \in \operatorname{Diff}^{1}\left(M^{2}\right)$ is topologically $\Omega$-stable, then int $\Omega(f)=\phi$ or $f$ is an Anosov diffeomorphism.

The main stage in proving Theorem 1 is the following. First we shall fix our notation.

Definition. For an open subset $U$ of $M$, we denote by $\mathscr{H}(U)$ the set of $f \in \operatorname{Diff}^{1}(M)$ whose periodic points in $U$ are all hyperbolic, and by $\mathscr{D}(U)$ the set of $f \in \operatorname{Diff}^{1}(M)$ whose periodic points are dense in $U$.

TheOREm 2. Let $M^{2}$ be a compact connected surface. Then for any open subset $U$ of $M^{2}$,

$$
\mathscr{D}(U) \cap \operatorname{int} \mathscr{H}(U) \subset \mathscr{D}\left(M^{2}\right) .
$$

Theorem 1 is proved in Section 2 and Theorem 2 in Section 4. Sections 3 and 5 are devoted to two propositions necessary for the proof of Theorem 2. In Appendix we shall prove a lemma about a non-transversal homoclinic point, which is necessary in Section 5 .

Throughout this paper except Appendix, ' $M$ ' will denote a compact connected surface without boundary.

I would like to thank Professor M. Adachi for his guidance of this 
area. I owe Professors G. Ikegami, M. Kurata and especially K. Shiraiwa many useful suggestions and criticisms.

\section{$\S 2$. Proofs of Theorem 1 and Corollary}

In this section we prove Theorem 1, assuming Theorem 2. We denote by $\mathscr{A}$ the set of all Anosov diffeomorphisms of $M$.

LEMMA 1. If $f \in \operatorname{int} \overline{\mathscr{D}(M)}$, then $f \in \overline{\mathscr{A}}$. Hence $\mathscr{A}$ is open and dense in int $\overline{\mathscr{D}(M)}$.

Proof. Let $f \in \operatorname{int} \overline{\mathscr{D}(M)}$. First, we suppose $f \notin \operatorname{int} \mathscr{H}(M)$. Some diffeomorphism $g$ near $f$ has a non-hyperbolic periodic point $p$. Since the dimension of $M$ is 2 , it is possible to make $p$ a sink or a source of a small $C^{1}$ perturbation $g_{1}$ of $g$, i.e., if $n$ is the period of $p$, then the eigenvalues of $T_{p} g_{1}^{n}$ have absolute values $<1$ (or $>1$ ). Obviously, $g_{1} \notin \overline{\mathscr{D}}(M)$. This contradicts the hypothesis, because $g_{1}$ can be chosen sufficiently near $f$. Thus $f \in \operatorname{int} \mathscr{H}(M)$. We can choose $f_{1} \in \operatorname{int} \mathscr{H}(M) \cap \mathscr{D}(M)$ near $f$. We here apply a theorem of Mañé [3], i.e. int $\mathscr{H}(M) \cap \mathscr{D}(M)=\mathscr{A}$ if the dimension of $M$ is 2 . Hence we have $f_{1} \in \mathscr{A}$. Therefore, $f \in \overline{\mathscr{A}}$.

q.e.d.

For each point $x \in M$, we define

$$
\mathscr{U}_{x}=\left\{f \in \operatorname{Diff}^{1}(M) ; x \notin \operatorname{int} \overline{\operatorname{Per}(f)}\right\} .
$$

Then we have

LEMma 2. If $f \notin \mathscr{U}_{x}$, then $f \in \mathscr{D}(M)$ or $f \in \overline{\operatorname{int} \mathscr{U}_{x}}$.

Proof. Let $f \notin \mathscr{U}_{x}$. By definition, $x \in \operatorname{int} \overline{\operatorname{Per}(f)}$. Let $U$ be a small neighborhood of $x$ in $\widehat{\operatorname{Per}(f)}$. When $f \in \operatorname{int} \mathscr{H}(U)$, by Theorem 2, we have $f \in \mathscr{D}(M)$. So it is sufficient to show that $f \in \overline{\operatorname{int} \mathscr{U}_{x}}$, when $f \notin \operatorname{int} \mathscr{H}(U)$. Then some $f_{1}$ near $f$ has a non-hyperbolic periodic point $p$ in $U$. Similarly, it is possible to make $p$ a sink or a source of some $C^{1}$ perturbation $f_{2}$ of $f_{1}$. Since $U$ is a small neighborhood of $x$, we can choose $h \in \operatorname{Diff}^{1}(M)$ with $h(x)=p$ in a small $C^{1}$ neighborhood of the identity of $M$. Put $g=$ $h^{-1} \cdot f_{2} \cdot h$. Clearly $g$ is $C^{1}$ near $f$. Naturally $x=h^{-1}(p)$ is a sink or source of $g$. Hence, for any $g_{1} \in \operatorname{Diff}^{1}(M)$ near $g$ we have $x \notin \operatorname{int} \overline{\operatorname{Per}\left(g_{1}\right)}$, or $g_{1} \in$ $\mathscr{U}_{x}$. This implies $g \in \operatorname{int} \mathscr{U}_{x}$. Since $g$ is near $f$, it follows that $f \in \overline{\text { int } \mathscr{U}_{x}}$.

$$
\text { q.e.d. }
$$

LEMMA 3. int $\mathscr{U}_{x} \cup \operatorname{int} \overline{\mathscr{D}(M)}$ is dense in $\operatorname{Diff}^{1}(M)$.

Proof. Suppose $f \notin \overline{\operatorname{int} \mathscr{U}_{x}}$. It suffices to show $f \in \overline{\mathscr{D}(M)}$. When $f \notin \mathscr{U}_{x}$, 
by Lemma 2, we have $f \in \mathscr{D}(M)$. When $f \in \mathscr{U}_{x}$ hence $f \in \mathscr{U}_{x}-\overline{\operatorname{int} \mathscr{U}_{x}}$, there is a sequence $f_{n} \notin \mathscr{U}_{x} \cup \overline{\text { int } \mathscr{U}_{x}}$ converging to $f$. By Lemma 2, $f_{n} \in \mathscr{D}(M)$. Hence $f \in \overline{\mathscr{D}}(M)$ follows.

q.e.d.

Now Theorem 1 is proved as follows: By Lemmas 1 and 3, $\mathscr{U}_{x} \cup \mathscr{A}$ is generic in Diff ${ }^{1}(M)$. Really it contains an open dense subset of $\operatorname{Diff}^{1}(M)$. By the Pugh's density theorem, the set

$$
\mathscr{C}=\left\{f \in \operatorname{Diff}^{1}(M) ; \Omega(f)=\overline{\operatorname{Per}(f)}\right\}
$$

is generic. Let $K$ be a dense countable subset of $M$. Then

$$
\begin{aligned}
\mathscr{B} & =\bigcap_{x \in R}\left(\mathscr{U}_{x} \cup \mathscr{A}\right) \cap \mathscr{C} \\
& =\left(\left(\bigcap_{x \in K} \mathscr{U}_{x}\right) \cap \mathscr{C}\right) \cup \mathscr{A}
\end{aligned}
$$

is generic in $\operatorname{Diff}^{1}(M)$. Now we need only check that if $f \in\left(\cap_{x \in K} \mathscr{U}_{x}\right) \cap \mathscr{C}$ then int $\Omega(f)=\phi$. From $f \in \bigcap_{x \in K} \mathscr{U}_{x}$, we have int $\overline{\operatorname{Per}(f)} \cap K=\phi$. But, since $K$ is dense in $M$, int $\overline{\operatorname{Per}(f)}=\phi$. On the other hand, $f \in \mathscr{C}$ means $\overline{\operatorname{Per}(f)}=\Omega(f)$. Hence int $\Omega(f)=\phi$ follows.

q.e.d.

Proof of Corollary. Let $f \in \operatorname{Diff}^{1}(M)$ be topologically $\Omega$-stable. First suppose $f \notin \mathscr{A}$. By Theorem 1, there is $g \in \operatorname{Diff}^{1}(M)$ near $f$ such that int $\Omega(g)=\phi$. By stability, it follows from the theorem of domain invariance that int $\Omega(f)=\phi$.

Next suppose $f \in \overline{\mathscr{A}}$. There is $f_{1} \in \mathscr{A}$ near $f$. Since $\Omega\left(f_{1}\right)=M$ ([9], p. 89), by stability, we have $\Omega(f)=M$. Hence by stability, $\Omega(g)=M$ for all $g$ near $f$. By Mañé [3], it follows that $f$ is Anosov.

q.e.d.

\section{§3. Laminations}

In this section we prepare a proposition for the proof of Theorem 2 . Let us begin with definitions.

Definition. Let $f \in \operatorname{Diff}^{1}(M)$. For a hyperbolic periodic point $p$ of $f$, we denote by $W^{s}(p ; f)$ (resp. $\left.W^{u}(p ; f)\right)$ the stable (resp. unstable) manifold of $f$ at $p$. We define $E^{s}(p ; f)$ to be the tangent space of $W^{s}(p ; f)$ at $p$. Likewise $E^{u}(p ; f)$ is defined.

In what follows, we shall drop ' $f$ ' in these symbols when it does not give rise to confusion.

Definition. A hyperbolic periodic point is called a saddle if it is not a sink nor source. We denote by $\operatorname{Sd}(f)$ the set of all saddles of $f$. 
Definition. $A C^{1}$ lamination of $M$ is a continuous foliation whose leaves are $C^{1}$ immersed submanifolds such that their tangent spaces, as a whole, form a continuous subbundle of $T M$.

Refer to $[1, \S 7]$ for general definitions.

We shall prove the following.

Proposition 1. Let $f \in \operatorname{Diff}^{1}(M)$. Let $U$ be an open subset of $M$ such that:

(1) $U$ is invariant under $f$.

(2) The periodic points in $U$ are all saddles and are dense in $U$.

(3) There is a continuous splitting $E^{s} \oplus E^{u}$ of $T M \mid U$ whose splitting at $\forall p \in \operatorname{Sd}(f) \cap U$ is $E^{s}(p ; f) \oplus E^{u}(p ; f)$.

Then there is an f-invariant $C^{1}$ lamination $W^{s}$ on $U$ such that (a) all laminae are tangent to $E^{s}$, (b) stable manifolds $W^{s}(p ; f), \forall p \in \operatorname{Sd}(f) \cap U$, are its laminae. Likewise there is an f-invariant lamination $W^{u}$ on $U$ with the corresponding properties.

Proof. We want to construct a lamination on a neighborhood of $\forall x_{0}$ $\in U$. First, we take a coordinate neighborhood $(Q, \varphi)$ of $x_{0}$ with the following properties.

(4) $Q \subset U$.

(5) $\varphi(Q)=[-1,1] \times[-1,1]$.

(6) $\varphi\left(x_{0}\right)=(0,0)$.

(7) Identify $Q$ with $[-1,1] \times[-1,1]$ and $E^{s}$ with $T \varphi\left(E^{s}\right)$. There is a $C^{0}$ map $w: Q \rightarrow R$ such that $|w(x)|<1 / 4$, and the vector $(1, w(x)$ ) spans $E^{s}(x), \forall x \in Q . \quad E^{s}(x)$ is the fiber of $E^{s}$ at $x$.

We, first of all, notice that stable manifolds $W^{s}(p), \forall p \in \operatorname{Sd}(f) \cap U$ are tangent to $E^{s}$. Because, if at a point $x \in W^{s}(p), E^{s}(x)$ is not tangent to $W^{s}(p)$, then $E^{s}\left(f^{\alpha n}(x)\right)=T f^{\alpha n}\left(E^{s}(x)\right)\left(\alpha\right.$ is the period of $p$ ) tends to $E^{u}(p)$ as $n \rightarrow \infty$ by hyperbolicity of $T_{p} f^{\alpha}$, contradicting continuity of $E^{s}$. Likewise unstable manifolds $W^{u}(p), \forall p \in \operatorname{Sd}(f) \cap U$, are tangent to $E^{u}$.

Let $\pi_{1}: Q \rightarrow[-1,1]$ be the projection on the first factor. Write $Q_{1}=$ $[-1,1] \times[-1 / 2,1 / 2] \subset Q$. For $\forall p \in \operatorname{Sd}(f) \cap Q$, let $K_{p}$ be the connected component of $W^{s}(p) \cap Q$ containing $p$. Let $h_{p}: K_{p} \rightarrow[-1,1]$ be the mapping defined by

$$
h_{p}(x)=\pi_{1}(x), \quad \forall x \in K_{p} .
$$

We want to show that $h_{p}$ is a homeomorphism if $p \in \operatorname{Sd}(f) \cap Q_{1}$. 
First, $h_{p}$ is one to one, because $K_{p}$ is an integral curve of the vector field $x \mapsto(1, w(x)), \forall x \in Q$, which spans $E^{s}$ over $Q$. So we show $h_{p}$ is onto. We notice that $K_{p}$ cannot meet the top nor the bottom of $Q$, because the slope of $K_{p}$ is less than $1 / 4$. So $h_{p}$ not being onto implies $\bar{K}_{p}-K_{p} \neq \phi$. Let $q \in \bar{K}_{p}-K_{p}$. See the figure.

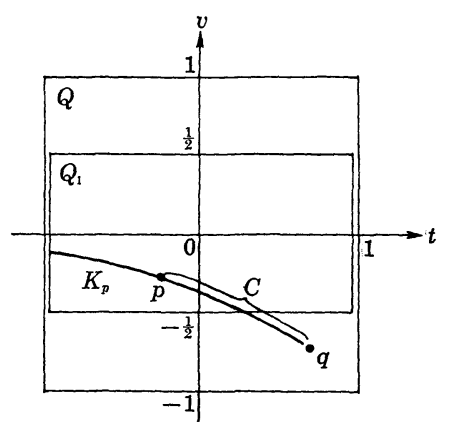

Thus $K_{p}$ includes one of the components of $W^{s}(p)-\{p\}$, say $C$. Since $f^{2 \alpha}(C)=C$, clearly we have $f^{2 \alpha}(q)=q$, namely $q \in \operatorname{Per}(f)$. Hence, by (2), $q \in \operatorname{Sd}(f)$. For $\forall x \in C, f^{-2 \alpha n}(x)$ tends to $q$ as $n \rightarrow \infty$. This implies $C \subset$ $W^{u}(q)$. Thus $C$ is tangent to $E^{s}$ and $E^{u}$ at once, which contradicts (3). Hence $h_{p}$ must be onto.

We denote by $\Pi$ the set of all $p \in \operatorname{Sd}(f) \cap Q$ such that $h_{p}$ is onto. By the above $\operatorname{Sd}(f) \cap Q_{1} \subset \Pi$. Let $\pi_{2}: Q \rightarrow[-1,1]$ be the projection on the second factor. When we put $V_{0}=\left\{\pi_{2} h_{p}^{-1}(0) ; p \in \Pi\right\} \subset[-1,1]$, it is easy to see that $V_{0}$ is dense in $[-1 / 2,1 / 2]$. For $\forall p \in \Pi$, we write $k_{u}=\pi_{2} \cdot h_{p}^{-1}$, where $u=\pi_{2} \cdot h_{p}^{-1}(0)$. Hence graph $\left(k_{u}\right)=K_{p}$. We define a function $v=$ $k(t, u), t \in[-1,1], u \in[-1 / 2,1 / 2]$ by the following:

$$
k(t, u)=\lim _{u^{\prime} \rightarrow u} k_{u^{\prime}}(t), \quad u^{\prime} \in V_{0} .
$$

The aim of the following is to prove that curves $t \mapsto(t, k(t, u)), u \in$ $[-1 / 2,1 / 2]$, are $C^{1}$ differentiable and tangent to $E^{s}$, and they form, as a whole, a $C^{1}$ lamination on a neighborhood of $x_{0}$.

1. $k(t, u)$ is well-defined: Let $(t, u)$ be fixed. Take $u_{1}, u_{2} \in V_{0}$ with $u_{1}<u<u_{2}$. If $p \in \operatorname{Sd}(f) \cap Q$ is in the domain between $\operatorname{graph}\left(k_{u_{1}}\right)$ and graph $\left(k_{u_{2}}\right)$, then $p$ belongs to $\Pi$. This is proved by the method proving in the above that $h_{p}$ is onto, and by the fact that subarcs $K_{p}, K_{q}$ of different two stable manifolds never meet each other. Remark that this fact also plays an important role in the following. 
So it is obvious that $\left\{K_{p} ; p \in \Pi\right\}$ meet the vertical segment $\{t\} \times$ $\left[k_{u_{1}}(t), k_{u_{2}}(t)\right] \subset Q$ densely. That is, the set $\left\{k_{u^{\prime}}(t) ; u^{\prime} \in V_{0}\right\}$ is dense in $\left[k_{u_{1}}(t), k_{u_{2}}(t)\right]$. Therefore, given $\varepsilon>0$, there is a finite sequence of numbers $u_{1}^{\prime}, u_{2}^{\prime}, \cdots, u_{n}^{\prime} \in V_{0}$ such that

(8) $u_{1}=u_{1}^{\prime}<u_{2}^{\prime}<\cdots<u_{n}^{\prime}=u_{2}$,

(9) $k_{u_{i+1}}^{\prime}(t)-k_{u_{i}}^{\prime}(t)<\varepsilon, \forall 1 \leq i<n$.

Let $j$ be the suffix with $u_{j}^{\prime}<u<u_{j+1}^{\prime}$. By (9), for $\forall u^{\prime}, u^{\prime \prime} \in V_{0} \cap$ $\left[u_{j}^{\prime}, u_{j+1}^{\prime}\right]$

$$
\left|k_{u^{\prime}}(t)-k_{u^{\prime \prime}}(t)\right|<k_{u_{j+1}^{\prime}}(t)-k_{u^{\prime}}(t)<\varepsilon .
$$

Hence $\left\{k_{u^{\prime}}(t) ; u^{\prime} \rightarrow u, u^{\prime} \in V_{0}\right\}$ is a Cauchy sequence.

q.e.d.

2. The convergence $k_{u^{\prime}}(t) \rightarrow k(t, u)$ is $C^{0}$ uniform: Given $\varepsilon>0$, choose a finite sequence of numbers $t_{1}, t_{2}, \cdots, t_{n} \in[-1,1]$ such that

(10) $-1=t_{1}<t_{2}<\cdots<t_{n}=1$,

(11) $t_{i+1}-t_{i}<\varepsilon / 2, \forall 1 \leq i<n$.

We can take $u_{1}, u_{2} \in V_{0}$ such that

(12) $u_{1}<u<u_{2}$

(13) $k_{u_{2}}\left(t_{i}\right)-k_{u_{1}}\left(t_{i}\right)<\varepsilon, \forall 1 \leq i \leq n$.

By the way, if $\left|t-t_{i}\right|<\varepsilon$, by (7) we have

$$
\begin{aligned}
\left|k_{u_{1}}(t)-k_{u_{1}}\left(t_{i}\right)\right| & =\left|\int_{t_{i}}^{t} \frac{d}{d t} k_{u_{1}}(t) d t\right| \\
& =\left|\int_{t_{i}}^{t} w\left(t, k_{u_{1}}(t)\right) d t\right| \\
& \leq\left|t-t_{i}\right| / 4<\varepsilon / 4 .
\end{aligned}
$$

Likewise $\left|k_{u_{2}}(t)-k_{u_{2}}\left(t_{i}\right)\right|<\varepsilon / 4$. Let $u^{\prime} \in V_{0}, u_{1}<u^{\prime}<u_{2}$. For $\forall t \in[-1,1]$, choose $t_{i}$ with $\left|t_{i}-t\right|<\varepsilon$. Then

$$
\begin{aligned}
\left|k(t, u)-k_{u^{\prime}}(t)\right| \leq & k_{u_{2}}(t)-k_{u_{1}}(t) \\
\leq & \left|k_{u_{2}}(t)-k_{u_{2}}\left(t_{i}\right)\right|+\left|k_{u_{2}}\left(t_{i}\right)-k_{u_{1}}\left(t_{i}\right)\right| \\
& +\left|k_{u_{1}}(t)-k_{u_{1}}\left(t_{i}\right)\right|<\varepsilon / 4+\varepsilon / 2+\varepsilon / 4=\varepsilon .
\end{aligned}
$$

Thus we have $\left|k(\cdot, u)-k_{u^{\prime}}(\cdot)\right|<\varepsilon$ if $u^{\prime} \in V_{0},\left|u^{\prime}-u\right|<\delta$, where $\delta=$ $\min \left\{\left|u_{1}-u\right|,\left|u_{2}-u\right|\right\}$. q.e.d.

3. $\left\{(d / d t) k_{u^{\prime}} ; u^{\prime} \rightarrow u, u^{\prime} \in V_{0}\right\}$ is uniformly convergent: Because

$$
\frac{d}{d t} k_{u^{\prime}}(t)=w\left(t, k_{u^{\prime}}(t)\right)
$$


and $k_{u}(t)$ is uniformly convergent.

q.e.d.

Therefore, $v=k(t, u),(t, u) \in[-1,1] \times[-1 / 2,1 / 2]$, is $C^{1}$ differentiable in $t$ and satisfies the differential equation $d v / d t=w(t, v)$.

It is easy to see that the mapping $H:[-1,1] \times[-1 / 2,1 / 2] \rightarrow Q$ defined by $H(t, u)=(t, k(t, u))$ is a homeomorphism (into). So we can define a $C^{1}$ lamination on a neighborhood of $x_{0}$ by letting its laminae be curves $t \mapsto$ $H(t, u), u \in[-1 / 2,1 / 2]$. To guarantee the existence of a global lamination $W^{s}$ on $U$, we need only check that two local laminations thus defined are always consistent with each other. But, otherwise, there must be a pair of stable manifolds having an intersection by the construction of laminae.

Clearly the lamination $W^{s}$ satisfies the desired conditions. q.e.d.

\section{§4. Theorem 2}

For simplicity we denote by $U_{f}$ the $f$ orbit of $U \subset M$. The following proposition plays a basic role in proving Theorem 2 .

Proposition 2. Let $U$ be an open subset of $M$. If $f \in \operatorname{int} \mathscr{H}(U)$, then there is a continuous splitting $E^{s} \oplus E^{u}$ of $T M \mid \overline{\mathrm{Sd}}(f) \cap U_{f}$ whose splitting at $\forall p \in \operatorname{Sd}(f) \cap U_{f}$ is $E^{s}(p ; f) \oplus E^{u}(p ; f)$.

The proof will be given in the next section. Now we prove Theorem 2.

TheOREM 2. For any open subset $U$ of $M$, we have

$$
\mathscr{D}(U) \cap \operatorname{int} \mathscr{H}(U) \subset \mathscr{D}(M) \text {. }
$$

Proof. Let $f \in \mathscr{D}(U) \cap \operatorname{int} \mathscr{H}(U)$. Clearly $\operatorname{Per}(f) \cap U_{f} \subset \operatorname{Sd}(f)$. So, Sd $(f)$ is dense in $U_{f}$. Applying Proposition 2, we have a splitting $E^{s} \oplus$ $E^{u}$ of $T M \mid \bar{U}_{f}$ whose splitting at $\forall p \in \operatorname{Sd}(f) \cap U_{f}$ is $E^{s}(p ; f) \oplus E^{u}(p ; f)$. Hence, by Proposition 1 , there are $f$-invariant laminations $W^{s}$ and $W^{u}$ such that $W^{s}(p ; f)$ and $W^{u}(p ; f), \forall p \in \operatorname{Sd}(f) \cap U_{f}$, are respectively their laminae.

It is sufficient to show $\bar{U}_{f}=M$, because $\operatorname{Per}(f)$ is dense in $U_{f}$. For this, we need only prove that for $\forall x_{0} \in \bar{U}_{f}$, there is a neighborhood of $x_{0}$ included in $\bar{U}_{f}$. Let us write $\Sigma=\operatorname{Sd}(f) \cap U_{f}$. We claim

(1) Let $p \in \Sigma$. Let $\varphi: R \rightarrow W^{s}(p), \varphi(0)=p$, be a parametrization of $W^{s}(p)$. Then $\varphi(\infty)=\lim _{t \rightarrow \infty} \varphi(t)$ never exists.

Proof of (1). Suppose there exists $\varphi(\infty)$. Let $\alpha$ be the period of $p$. First, $\varphi(\infty) \notin U_{f}$, because by Proposition $1 W^{s}(p)$ is a lamina of $W^{s}$. It is 
also clear that $f^{2 \alpha}(\varphi(\infty))=\varphi(\infty)$. Since the laminations $W^{s}, W^{u}$ are transversal, we have $q \in \Sigma$ with $\varphi\{(0, \infty)\} \cap W^{u}(q) \neq \phi$. Let $y \in \varphi\{(0, \infty)\} \cap$ $W^{u}(q)$. Denote by $\beta$ the period of $q$. Since $y \in W^{u}(q), f^{-2 \alpha \beta n}(y) \rightarrow q$ as $n \rightarrow \infty$. Since $y \in \varphi\{(0, \infty)\}, f^{-2 \alpha \beta n}(y) \rightarrow \varphi(\infty)$ as $n \rightarrow \infty$. Hence $q=\varphi(\infty)$. This is a contradiction, because $\varphi(\infty) \notin U_{f}$. q.e.d.

By continuity of $E^{s} \oplus E^{u}$, we may choose a coordinate neighborhood $(Q, \psi)$ of $x_{0}$ satisfying the following $(2) \sim(4)$.

(2) $\psi(Q)=[-1,1] \times[-1,1]$

(3) $\psi\left(x_{0}\right)=(0,0)$

(4) Identify $Q$ with its image by $\psi$ and $E^{s}, E^{u}$ with $T \psi\left(E^{s}\right), T \psi\left(E^{u}\right)$ respectively. Then we have $C^{0}$ functions $w_{s}, w_{x}: Q \cap \bar{U}_{f} \rightarrow[-1 / 4,1 / 4]$ such that $\left(1, w_{s}(x)\right),\left(w_{u}(x), 1\right) \in T_{x} Q$ span respectively $E^{s}(x), E^{u}(x)$ for $\forall x \in Q \cap \bar{U}_{f}$.

Let $p \in \Sigma \cap Q$. We denote by $K_{p}^{s}$ (resp. $K_{p}^{u}$ ) the connected component of $W^{s}(p) \cap Q$ (resp. $W^{u}(p) \cap Q$ ) containing $p$. We express the coordinate system in $Q$ as $(t, v)$. Noting that $K_{p}^{s}$ is an integral curve of the vector field $x \mapsto\left(1, w_{s}(x)\right)\left(x \in Q \cap U_{f}\right)$, we have a function $v=k_{p}(t)$ with graph $\left(k_{p}\right)$ $=K_{p}^{s}$. Let $I I$ be the set of all $p \in \Sigma \cap Q$ such that the domain of $k_{p}$ is $[-1,1]$. Put $Q_{1}=[-1,1] \times[-1 / 2,1 / 2] \subset Q$. As in the previous section, we can prove $\Sigma \cap Q_{1} \subset \Pi$ by virtue of (1).

Let us fix a point $p_{0} \in[-1 / 4,1 / 4] \times[-1 / 4,1 / 4] \cap \Sigma$. Similarly as above, we have a function $t=h(v), v \in[-1,1]$ with graph $(h)=K_{p_{0}}^{u}$. For $\forall p \in \Pi$, $K_{p}^{s} \cap K_{p_{0}}^{u}$ consists of just a point. Let $\pi_{2}(t, v)=v$ be the projection. Define $V_{0}=\left\{\pi_{2}\left(K_{p}^{s} \cap K_{p_{0}}^{u}\right) ; p \in \Pi\right\}$. Since $\Sigma \cap Q_{1} \subset \Pi, V_{0}$ is dense in [-1/2,1/2]. For $\forall u^{\prime} \in V_{0}$, we put $k\left(t, u^{\prime}\right)=k_{p}(t)$, where $\pi_{2}\left(K_{p}^{s} \cap K_{p_{0}}^{u}\right)=u^{\prime}$. See the figure.

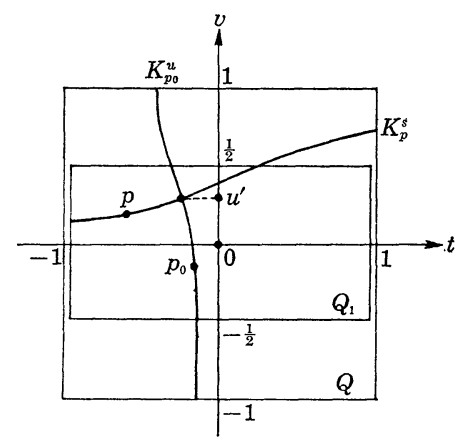

Now we define a function $v=k(t, u),(t, u) \in[-1,1] \times[-1 / 2,1 / 2]$ by

$$
\underline{k}(t, u)=\lim _{u^{\prime} \uparrow u} k\left(t, u^{\prime}\right), \quad u^{\prime} \in V_{0} .
$$


First, this is well-defined, because $k\left(t, u^{\prime}\right)$ is monotonuous in $u^{\prime} \in V_{0}$. As in the previous section, we have similarly that this convergence is $C^{1}$ uniform in $t \in[-1,1]$.

Likewise we define another function $v=\bar{k}(t, u),(t, u) \in[-1,1] \times$ $[-1 / 2,1 / 2]$ by

$$
\bar{k}(t, u)=\lim _{u^{\prime} \downarrow u} k\left(t, u^{\prime}\right), \quad u^{\prime} \in V_{0} .
$$

We want to show $\underline{k}=\bar{k}$. Suppose that for some $t_{1}, u_{1} \underline{k}\left(t_{1}, u_{1}\right) \neq \bar{k}\left(t_{1}, u_{1}\right)$. Let $D$ be the region in $Q$ between the graphs of $k\left(\cdot, u_{1}\right)$ and $\bar{k}\left(\cdot, u_{1}\right)$. First we have $D \cap U_{f}=\phi$. If not, we can take two points $p_{1}, p_{2} \in \Sigma \cap D$. By (1), they belong to $\Pi$. So the region in $Q$ between $K_{p_{1}}^{s}$ and $K_{p_{2}}^{s}$ is included in $D$. But this is impossible, because $\underline{k}\left(t_{2}, u_{1}\right)=\bar{k}\left(t_{2}, u_{1}\right)$ where $\left(t_{2}, u_{1}\right) \in K_{p_{0}}^{u}$. Thus $D \cap U_{f}=\phi$.

We also have $D \cap U_{f} \neq \phi$. This is shown as follows. Put $x_{1}=$ $\left(t_{1}, k\left(t_{1}, u_{1}\right)\right)$. We notice that the graphs of $k\left(\cdot, u^{\prime}\right), u^{\prime} \in V_{0}$, are included in $U_{f}$. So, $x_{1}=\lim \left(t_{1}, k\left(t_{1}, u^{\prime}\right)\right)\left(u^{\prime} \uparrow u_{1}, u^{\prime} \in V_{0}\right)$ is contained in $\bar{U}_{f}$. Hence we can choose a point $p \in \Sigma$ near $x_{1}$. Then $K_{p}^{u}$ meets the graph of $\underline{k}\left(\cdot, u_{1}\right)$ at a point near $x_{1}$. So it meets $D$, too. Since $K_{p}^{u} \subset U_{f}$, we have $D \cap$ $U_{f} \neq \phi$.

Thus we have a contradiction. Therefore, $k=\bar{k}$. Hereafter we write $k=\underline{k}=\bar{k}$.

It is easily shown that the mapping $H:[-1,1] \times[-1 / 2,1 / 2] \rightarrow Q$ defined by $H(t, u)=(t, k(t, u))$ is a homeomorphism (into). Moreover, its image is in $\bar{U}_{f}$. So it is sufficient to show that $\operatorname{Im}(H) \supset[-1 / 2,1 / 2] \times[-1 / 4,1 / 4]$.

By (4), $K_{p_{0}}^{u}$ meets the segments $[-1 / 2,1 / 2] \times\{1 / 2\}$, and $[-1 / 2,1 / 2] \times$ $\{-1 / 2\} \subset Q$. Let these intersections be $y_{1}, y_{2}$ respectively. By definition, $\operatorname{graph}(k(\cdot, 1 / 2))$ goes through $y_{1}$, and $\operatorname{graph}(k(\cdot, 1 / 2))$ through $y_{2}$. Hence it follows from $|(\partial / \partial t) k(t, u)|=\mid w_{s}(t, k(t, u) \mid<1 / 4$ that for $\forall t \in[-1 / 2,1 / 2]$, $k(t, 1 / 2)>1 / 4$ and $k(t,-1 / 2)<-1 / 4$. Hence, as $u$ goes from $-1 / 2$ to $1 / 2$ with $t \in[-1 / 2,1 / 2]$ fixed, $k(t, u)$ varies from $k(t,-1 / 2)<-1 / 4$ to $k(t, 1 / 2)$ $>1 / 4$. By continuity of $k$, it follows that for $\forall t \in[-1 / 2,1 / 2],\{t\} \times[-1 / 4$, $1 / 4] \subset \operatorname{Im}(H)$. That is, $[-1 / 2,1 / 2] \times[-1 / 4,1 / 4] \subset \operatorname{Im}(H)$. Hence $x_{0}=$ $(0,0) \in \operatorname{int} \bar{U}_{f}$.

Thus we have proved Theorem 2.

q.e.d.

\section{§5. Proposition 2}

In the proof of Theorem 2, Proposition 2 still remains to be proved. 
Proposition 2. Let $U$ be an open subset of $M$. If $f \in \operatorname{int} \mathscr{H}(U)$, then there is a continuous splitting $E^{s} \oplus E^{u}$ of $T M \mid \overline{\operatorname{Sd}(f) \cap \bar{U}_{f}}$ whose splitting at $\forall p \in \operatorname{Sd}(f) \cap U_{f}$ is $E^{s}(p ; f) \oplus E^{u}(p ; f)$.

Proof. We state two assertions, which will be proved later, and using them, we obtain the proof of Proposition 2.

Let $G M$ be the bundle over $M$ whose fiber at $x$ consists of all 1dimensional subspaces of $T_{x} M$. Let $d$ be the metric on $G M$ induced from a Riemann metric on $M$.

Assertion 1. There is a $C^{1}$ neighborhood $\mathscr{U}$ of $f$ such that

$$
\inf \left\{d\left(E^{s}(p ; g), E^{u}(p ; g)\right) ; g \in \mathscr{U}, p \in \operatorname{Sd}(g) \cap U_{g}\right\}>0 .
$$

Assertion 2. There is a positive integer $\nu$ such that

$$
\left\|T f^{\nu}\left|E^{s}(p)\|/\| T f^{\nu}\right| E^{u}(p)\right\| \leq 1 / 2, \quad \forall p \in \operatorname{Sd}(f) \cap U_{f} .
$$

Now Proposition 2 is proved as follows: Let $x \in \overline{\operatorname{Sd}(f) \cap U_{f}}$. Let $p_{n}, q_{n} \in \operatorname{Sd}(f) \cap U_{f}, n=1,2, \cdots$ be two sequences converging to $x$ such that $E^{s}\left(p_{n}\right), E^{u}\left(p_{n}\right) ; E^{s}\left(q_{n}\right), E^{u}\left(q_{n}\right)$ have a limit. Denote their limits by $F^{s}$, $F^{u} ; G^{s}, G^{u}$ respectively. It is sufficient to prove $F^{s}=G^{s}$ and $F^{u}=G^{u}$. Suppose $F^{s} \neq G^{s}$, for example. It follows from Assertion 1 that $F^{s} \neq F^{u}$, $G^{s} \neq G^{u}$. Our argument is divided into three cases.

1. The case $F^{s} \neq G^{u}$. It follows from Assertion 2 that

$$
\left\|T_{x} f^{k \nu}\left|F^{s}\|/\| T_{x} f^{k \nu}\right| F^{u}\right\| \leq 1 / 2^{k}, \quad \forall k>0 .
$$

Since $G^{s} \neq F^{s}$ and $G^{u} \neq F^{s}$, we have by this that given $\varepsilon>0$, there is $k>0$ such that

$$
\begin{aligned}
& d\left(T_{x} f^{k \nu}\left(G^{s}\right), T_{x} f^{k \nu}\left(F^{u}\right)\right)<\varepsilon, \\
& d\left(T_{x} f^{k \nu}\left(G^{u}\right), T_{x} f^{k \nu}\left(F^{u}\right)\right)<\varepsilon .
\end{aligned}
$$

Hence we have

$$
d\left(T_{x} f^{k \nu}\left(G^{s}\right), T_{x} f^{k \nu}\left(G^{u}\right)\right)<2 \varepsilon .
$$

This clearly contradicts Assertion 1.

2. The case $F^{u} \neq G^{s}$. This is the same with the case 1 , if $F$ and $G$ are interchanged.

3. The case $F^{s}=G^{u}$ and $F^{u}=G^{s}$. By Assertion 2, we have 


$$
\begin{aligned}
\left\|T_{x} f^{\nu}\left|F^{s}\|/\| T_{x} f^{\nu}\right| F^{u}\right\| & \leq 1 / 2, \\
\left\|T_{x} f^{\nu}\left|G^{s}\|/\| T_{x} f^{\nu}\right| G^{u}\right\| & \leq 1 / 2 .
\end{aligned}
$$

The above inequalities contradict each other, because $F^{s}=G^{u}$ and $F^{u}=G^{s}$. Thus we have derived a contradiction from the assumption $F^{s} \neq G^{s}$. Hence we have Proposition 2. q.e.d.

To prove Assertions 1, 2 we prepare the following.

Assertion 3. For some small $C^{1}$ neighborhood $\mathscr{U}_{1}$ of $f$, there is a constant $0<\lambda<1$ such that for $\forall g \in \mathscr{U}_{1}, \forall p \in \operatorname{Sd}(g) \cap U_{g}$

$$
\begin{aligned}
& \left\|T g^{\alpha(p)} \mid E^{s}(p ; g)\right\|<\lambda, \\
& \left\|T g^{-\alpha(p)} \mid E^{u}(p ; g)\right\|<\lambda,
\end{aligned}
$$

where $\alpha(p)$ means the $g$ period of $p$.

Proof of Assertion 3. Suppose otherwise. We may assume without loss of generality that for any $\varepsilon>0$, there exists $g$ in the $\varepsilon-C^{1}$ neighborhood of $f$ with $\left\|\operatorname{Tg}^{\alpha(p)} \mid E^{s}(p ; g)\right\|>1-\varepsilon$ for some $p \in \operatorname{Sd}(g) \cap U$. Let $\varepsilon_{1}$ $=1-\left\|T g^{\alpha(p)} \mid E^{s}(p ; g)\right\|$. Clearly $0<\varepsilon_{1}<\varepsilon$.

By Lemma $\mathrm{B}_{2}$ in Appendix, we have a $C \varepsilon-C^{1}$ perturbation $h$ of the identity of $M$ ( $C$ is a constant as in that lemma) such that

(1) $h(p)=p$.

(2) $T_{p} h=\left(1-\varepsilon_{1}\right)^{-1} I_{p}$ where $I_{P}: T_{p} M \longleftarrow$ is the identity.

(3) $h(x)=x$ for $x$ outside a small neighborhood of $p$.

We define $g_{1}=h \cdot g \in \operatorname{Diff}^{1}(M)$. By (1), (3), $g_{1}=g$ on the orbit of $p$. Clearly $E^{s}(p ; g)$ is invariant under $T_{p} g^{\alpha}(p)$. But we have

$$
\begin{aligned}
\left\|T_{p} g_{1}^{\alpha(p)} \mid E^{s}(p ; g)\right\| & =\left\|T_{p} h \cdot T_{p} g^{\alpha(p)} \mid E^{s}(p ; g)\right\| \\
& =\left(1-\varepsilon_{1}\right)^{-1}\left\|T_{p} g^{\alpha(p)} \mid E^{s}(p ; g)\right\|=1 .
\end{aligned}
$$

Since the dimension of $E^{s}(p ; g)$ is one, it follows that $p$ is not hyperbolic for $g_{1}$. By construction, $g_{1}$ is near $f$ in $\operatorname{Diff}^{1}(M)$, so $f \notin \operatorname{int} \mathscr{H}(U)$. This is a contradiction.

Proof of Assertion 1. Suppose it is not true. Then, for any $\varepsilon>0$ we have $g \in \mathscr{U}_{1}$ with

$$
\tan d\left(E^{s}(p ; g), E^{u}(p ; g)\right)<2^{-1} \varepsilon(1-\lambda)
$$

for some $p \in \operatorname{Sd}(g) \cap U_{g}$, where $\mathscr{U}_{1}$ and $\lambda$ are the ones given in Assertion 3. Let $\alpha$ be the period of $p$. Take a small neighborhood $Q$ of $p$ with 
(1) $Q \nexists g^{n}(p), \forall 1 \leq n \leq \alpha-1$.

We denote by $W_{r}^{s}(p ; g)\left(W_{r}^{u}(p ; g)\right)$ the local stable (unstable) manifold of size $r>0$. We choose orthogonal coordinates $(t, v)$ in $Q$ with origin at $p$ such that the $t$-axis is $W_{r}^{s}(p ; g)$.

The function $v=\psi(t)$ representing $W_{r}^{u}(p ; g)$ has the form:

(2) $\psi(t)=c \cdot t+R(t), t \in(-r, r)$.

(3) $|c|<2^{-1} \varepsilon(1-\lambda)$.

(4) $R(0)=R^{\prime}(0)=0$.

By (4), taking $r$ small, we may assume:

(5) $\left|R^{\prime}(t)\right|<2^{-1} \varepsilon(1-\lambda), \forall t \in(-r, r)$.

So we have

(6) $|R(t)|<2^{-1} \varepsilon(1-\lambda) r, \forall t \in(-r, r)$.

Noting $p=g^{\alpha}(p)=(0,0)$, we define $C^{1}$ mappings $h_{1}, h_{2}:(-r, r) \rightarrow$ $(-r, r)$ respectively by

(7) $h_{1}(t)=\pi_{1} g^{\alpha}(t, 0)$

(8) $h_{2}(t)=\pi_{1} g^{-\alpha}(t, \psi(t))$, where $\pi_{1}$ is the projection on the first factor. Since $\left|h_{1}^{\prime}(0)\right|<\lambda,\left|h_{2}^{\prime}(0)\right|<\lambda$, by taking $r$ small enough we have

(9) $\left|h_{1}(t)\right| \leq \lambda|t|,\left|h_{2}(t)\right| \leq \lambda|t|, \forall t \in(-r, r)$.

Put $b=r / 2$ and $\delta=(1-\lambda) b$. For $\forall t \in(-r, r)$ we have

(10) $|\psi(t)| \leq|c| r+|R(t)|<\varepsilon r(1-\lambda)=2 \varepsilon \delta$,

(11) $\left|\psi^{\prime}(t)\right| \leq|c|+\left|R^{\prime}(t)\right|<\varepsilon(1-\lambda)<\varepsilon$.

Let $x_{1}=(b, 0), x_{2}=(b, \psi(b))$. Then

$$
\begin{aligned}
& \left|\pi_{1} g^{\alpha}\left(x_{1}\right)-b\right|=\left|h_{1}(b)-b\right|>b-\lambda b=\delta, \\
& \left|\pi_{1} g^{\alpha}\left(x_{2}\right)-b\right|=\left|h_{2}(b)-b\right|>b-\lambda b=\delta .
\end{aligned}
$$

Hence we have

(12) $\left\|g^{\alpha}\left(x_{1}\right)-x_{1}\right\|>\delta$

(13) $\left\|g^{-\alpha}\left(x_{2}\right)-x_{1}\right\|>\delta$.

We define a $C^{1}$ mapping $k: Q \rightarrow Q$ as follows: Let $\phi: R \rightarrow \boldsymbol{R}$ be a $C^{1}$ function with $\phi(-\infty, 1 / 2]=1, \phi[1, \infty)=0$.

(14) $k(t, v)=\left(t, v-\phi\left(\left\{(t-b)^{2}+v^{2}\right\} / \delta^{2}\right) \cdot \psi(t)\right)$.

Then the following holds:

(15) $k(t, \psi(t))=(t, 0)$, if $|t-b|$ is sufficiently small.

(16) $k(x)=x$, if $\left\|x-x_{1}\right\|>\delta$.

(17) $k$ is near the identity of $Q$ in the $C^{1}$ sense when $\varepsilon$ is small.

The last is shown as follows. By (10), (17) is true in the $C^{0}$ sense. By (10), (11) and the fact that $\phi\left(\left\{(t-b)^{2}+v^{2}\right\} / \delta^{2}\right)=0$ if $|t-b|>\delta$, we have 


$$
\begin{aligned}
\left\|\frac{\partial}{\partial t}\{k(t, v)-(t, v)\}\right\| & =\mid \phi^{\prime}\left(\left\{(t-b)^{2}+v^{2}\right\} / \delta^{2}\right) \cdot 2 \delta^{-2}(t-b) \cdot \psi(t) \\
& \quad+\phi\left(\left\{(t-b)^{2}+v^{2}\right\} / \delta^{2}\right) \cdot \psi^{\prime}(t) \mid \\
& \leq\left|\phi^{\prime}\right| \cdot 2 \delta^{-2} \delta \cdot 2 \varepsilon \delta+|\phi| \varepsilon \\
& =\left(4\left|\phi^{\prime}\right|+|\phi|\right) \varepsilon \rightarrow 0 .
\end{aligned}
$$

We also have the same result about $\partial / \partial v$. Thus (17) follows.

We extend $k$ to a mapping of $M \rightarrow M$ by letting $k(x)=x$ for $x$ outside $Q$. By (17) we make $k$ a diffeomorphism of $M$. Then we define $g_{1}=k \cdot g$ $\in \operatorname{Diff}^{1}(M)$. By (1), (16), we have

(18) $g_{1}^{n}(p)=g^{n}(p), \forall n \in Z$.

In particular, $p$ is a periodic point of $g_{1}$.

By (1) and (16) we have

$$
g_{1}^{-\alpha}\left(x_{1}\right)=\left(g^{-1} k^{-1}\right)^{\alpha}\left(x_{1}\right)=g^{-\alpha}\left(x_{2}\right) .
$$

So it follows from (13), (9) that

$$
g_{1}^{-n \alpha}\left(g^{-\alpha}\left(x_{2}\right)\right)=g^{-n \alpha}\left(g^{-\alpha}\left(x_{2}\right)\right), \quad \forall n \geq 1 .
$$

Hence we have

(19) $g_{1}^{-n \alpha}\left(x_{1}\right)=g^{-n \alpha}\left(x_{2}\right), \forall n \geq 1$.

This implies that $x_{1} \in W^{u}\left(p ; g_{1}\right)$, because $g_{1}^{-n \alpha}\left(x_{1}\right)$ approaches $p$ as $n \rightarrow \infty$. By (15), we can prove similarly that any point of the form $(t, 0)$ with $|t-b|$ small enough is contained in $W^{u}\left(p ; g_{1}\right)$.

By (12) and (16), we have

$$
g_{1}^{\alpha}\left(x_{1}\right)=(k \cdot g)^{\alpha}\left(x_{1}\right)=k \cdot g^{\alpha}\left(x_{1}\right)=g^{\alpha}\left(x_{1}\right) \text {. }
$$

Similarly we have

(20) $g_{1}^{n \alpha}\left(x_{1}\right)=g^{n \alpha}\left(x_{1}\right), \forall n \geq 1$.

This implies that $x_{1} \in W^{s}\left(p ; g_{1}\right)$. Also, we can prove similarly that any point of the form $(t, 0)$ with $|t-b|$ small enough is contained in $W^{s}\left(p ; g_{1}\right)$.

Thus it is proved that $x_{1}$ is a non-transversal homoclinic point of $g_{1}$. It is clear that the $g_{1}$ orbit of $x_{1}$ meets $U$ and hence $g_{1}$ has a nontransversal homoclinic point in $U$. By Lemma $\mathrm{A}$ in Appendix, we have a small perturbation of $g_{1}$ with a non-hyperbolic periodic point in $U$. This contradicts the hypothesis, i.e. $f \in \operatorname{int} \mathscr{H}(U)$. q.e.d

For the proof of Assertion 2 we first prove the following. For $\forall p \in$ $\operatorname{Sd}(f) \cap U_{f}$, we define $N(p)$ to be the smallest positive integer $n$ such that 


$$
\left\|T f^{n}\left|E^{s}(p ; f)\|/\| T f^{n}\right| E^{u}(p ; f)\right\|<\lambda .
$$

Clearly, $N(p)$ does not exceed the period of $p$.

Assertion 4. $\sup \left\{N(p) ; p \in \operatorname{Sd}(f) \cap U_{f}\right\}<\infty$.

Proof. Given $\varepsilon>0$, take a positive integer $n_{0}$ such that

(1) $(1-\lambda)^{n_{0}}<\lambda \varepsilon^{2}$.

Suppose the above is not true. Then there is $p \in \operatorname{Sd}(f) \cap U_{f}$ with $N(p)$ $\geq n_{0}+3$. Let $\tau$ be the greatest integer such that $2 \tau+2 \leq N(p)$. Let $\alpha$ be the period of $p$. Then,

(2) $n_{0}+2 \leq 2 \tau+2 \leq N(p) \leq \alpha$.

We take unit vectors $V^{s} \in E^{s}(p ; f), V^{u} \in E^{u}(p ; f)$. In what follows, we simply write

(3) $p_{n}=f^{n-1}(p)$,

(4) $V_{n}^{s}=T f^{n-1}\left(V^{s}\right), V_{n}^{u}=T f^{n-1}\left(V^{u}\right), \forall n \in Z$.

Note that $p_{\alpha}=p_{0}$ but $V_{\alpha}^{s} \neq V_{0}^{s}, V_{\alpha}^{u} \neq V_{0}^{u}$.

By Lemma $\mathrm{B}_{2}$. in Appendix we construct $h=h_{s} \in \operatorname{Diff}^{1}(M)$ with the following properties (5) (11) in such a way that $h$ approaches the identity in the $C^{1}$ sense as $\varepsilon \rightarrow 0$.

(5) $h\left(p_{n}\right)=p_{n}, \forall 0 \leq n<\alpha$.

(6) $h(x)=x, \forall x$ outside a small neighborhood of $\left\{p_{n} ; 0 \leq n<\alpha\right\}$.

(7) $T_{p_{1}} h\left(V_{1}^{s}\right)=V_{1}^{s}, T_{p_{1}} h\left(V_{1}^{u}\right)=V_{1}^{u}+\varepsilon V_{1}^{s}$.

(8) $\forall 2 \leq n \leq \tau+1$;

$$
T_{p_{n}} h\left(V_{n}^{s}\right)=(1-\varepsilon)^{-1} V_{n}^{s}, \quad T_{p_{n}} h\left(V_{n}^{u}\right)=(1-\varepsilon) V_{n}^{u} .
$$

(9) $\forall \tau+2 \leq n \leq 2 \tau+1$;

$$
T_{p_{n}} h\left(V_{n}^{s}\right)=(1-\varepsilon) V_{n}^{s}, \quad T_{p_{n}} h\left(V_{n}^{u}\right)=(1-\varepsilon)^{-1} V_{n}^{u} .
$$

(10) $\forall 2 \tau+2 \leq n \leq \alpha-1 ; T_{p_{n}} h: T_{p_{n}} M \longleftarrow$ is the identity.

(11) $T_{p_{\alpha}} h\left(V_{\alpha}^{s}\right)=V_{\alpha}^{s}, T_{p_{\alpha}} h\left(V_{\alpha}^{u}\right)=V_{\alpha}^{u}-\varepsilon V_{\alpha}^{s}$.

Then we define $g=h \cdot f \in \operatorname{Diff}^{1}(M)$. By (5),

(12) $g^{n}(p)=f^{n}(p), \forall n \in Z$.

It follows from (8), (9), (10) that

(13) $T_{p_{1}} g^{n}=T_{p_{1}} f^{n}, \forall 2 \tau \leq n \leq \alpha-2$.

Now we want to show that

(14) $T_{p_{0}} g^{\alpha}=T_{p_{0}} f^{\alpha}$.

For this, it is sufficient to show the following:

(15) $T_{p_{0}} g\left(V_{0}^{s}\right)=V_{\alpha}^{s}, T_{p_{0}} g\left(V_{0}^{u}\right)=V_{\alpha}^{u}$. 
The first is easily shown, so we check the latter:

$$
\begin{aligned}
T_{p_{0}} g^{\alpha}\left(V_{0}^{u}\right) & =T_{p_{\alpha-1}} g T_{p_{1}} g^{\alpha-2} T_{p_{0}} g\left(V_{0}^{u}\right) & & \\
& =\left(T_{p_{\alpha}} h T_{p_{\alpha-1}} f\right) \cdot\left(T_{p_{1}} f^{\alpha-2}\right) \cdot\left(T_{p_{1}} h T_{p_{0}} f\right)\left(V_{0}^{u}\right) & & \text { (by (13)) } \\
& =T_{p_{\alpha}} h T_{p_{1}} f^{\alpha-1} T_{p_{1}} h\left(V_{1}^{u}\right) & & \\
& =T_{p_{\alpha}} h T_{p_{1}} f^{\alpha-1}\left(V_{1}^{u}+\varepsilon V_{1}^{s}\right) & & (\text { by (7)) } \\
& =T_{p_{\alpha}} h\left(V_{\alpha}^{u}+\varepsilon V_{\alpha}^{s}\right) & & \text { (by (4)) } \\
& =\left(V_{\alpha}^{u}-\varepsilon V_{\alpha}^{s}\right)+\varepsilon V_{\alpha}^{s} & & \text { (by (11)) } \\
& =V_{\alpha}^{u} . & &
\end{aligned}
$$

It follows from (14) that $g^{\alpha}$ is hyperbolic at $p_{0}$ and

(16) $E^{u}\left(p_{0} ; g\right)=E^{u}\left(p_{0} ; g\right)$.

It is also clear by the construction of $h$ that

(17) $E^{s}\left(p_{n} ; g\right)=E^{s}\left(p_{n} ; f\right), \forall 0 \leq n<\alpha$.

Now we are in a position to conclude the proof. We estimate $d\left(E^{s}\left(p_{r+1} ; g\right), E^{u}\left(p_{x+1} ; g\right)\right)$. By virtue of (16) and (17), this is equal to the angle $\theta$ between $T_{p_{0}} g^{\tau+1}\left(V_{0}^{u}\right)$ and $E^{s}\left(p_{\tau+1} ; f\right)$.

Write $T_{p_{0}} g^{\tau+1}\left(V_{0}^{u}\right)=\left(w_{s}, w_{u}\right)$ regarding $E^{s}\left(p_{\tau+1} ; f\right) \oplus E^{u}\left(p_{\tau+1} ; f\right)$. Let us compute $w_{s}, w_{u}$.

$$
\begin{aligned}
T_{p_{0}} g^{\tau+1}\left(V_{0}^{u}\right) & =T_{p} g^{\tau}\left(\varepsilon V_{1}^{s}+V_{1}^{u}\right) \quad(\text { by }(7)) \\
& =\varepsilon T_{p} g^{\tau}\left(V_{1}^{s}\right)+T_{p} g^{\tau}\left(V_{1}^{u}\right) \\
& =\varepsilon(1-\varepsilon)^{\tau} T_{p} f^{\tau}\left(V_{1}^{s}\right)+(1-\varepsilon)^{\tau} T_{p} f^{\tau}\left(V_{1}^{u}\right) .
\end{aligned}
$$

Hence

(18) $w_{s}=\varepsilon(1-\varepsilon)^{-\tau} T_{p} f^{\tau}\left(V_{1}^{s}\right), w_{u}=(1-\varepsilon)^{\tau} T_{p} f^{\tau}\left(V_{1}^{u}\right)$.

By (2) and the definition of $N(p)$, it follows that

$$
\begin{aligned}
\left\|w_{u}\right\| /\left\|w_{s}\right\|= & \varepsilon^{-1}(1-\varepsilon)^{2 \tau}\left\|T_{p} f^{\tau}\left(V_{1}^{u}\right)\right\| /\left\|T_{p} f^{\tau}\left(V_{1}^{s}\right)\right\| \quad \text { (by (18)) } \\
& <\varepsilon^{-1} \lambda^{-1}(1-\varepsilon)^{2 \tau} \\
& <\varepsilon^{-1} \lambda^{-1}(1-\varepsilon)^{n_{0}}<\varepsilon \quad \text { (by (1)) } .
\end{aligned}
$$

Hence it follows that

$$
\cos \theta=\left(w_{s}+w_{u}\right) \cdot w_{u} /\left\|w_{s}+w_{u}\right\|\left\|w_{u}\right\|>(1-\varepsilon) /(1+\varepsilon) \longrightarrow 1,
$$

as $\varepsilon \rightarrow 0$.

Therefore, $\theta$ approaches 0 as $\varepsilon \rightarrow 0$, which contradicts Assertion 1 . q.e.d.

Proof of Assertion 2. By Assertion 4, let 


$$
N=\sup \left\{N(p) ; p \in \operatorname{Sd}(f) \cap U_{f}\right\}<\infty .
$$

Put $C=\|T f\|\left\|T f^{-1}\right\|$. We take a positive integer $m$ with $C^{N} \lambda^{m}<1 / 2$. Let $\nu=(m+1) N$.

For $\forall p \in \operatorname{Sd}(f) \cap U_{f}$, we define $q_{1}, q_{2}, \cdots, q_{r+1} \in \operatorname{Sd}(f)$ as follows:

(1) $q_{1}=p$.

(2) $q_{i+1}=f^{N\left(q_{i}\right)}\left(q_{i}\right), 1 \leq i \leq r$.

(3) $\nu-N \leq \sum_{i=1}^{r} N\left(q_{i}\right)<\nu$.

Since $N\left(q_{i}\right) \leq N, \forall 1 \leq i \leq r$, it follows that $r N \geq \nu-N=m N$ and hence $r \geq m$.

Noting $E^{s}, E^{u}$ are 1 dimensional, we have

$$
\begin{aligned}
\frac{\left\|T f^{\nu} \mid E^{s}(p)\right\|}{\left\|T f^{\nu} \mid E^{u}(p)\right\|} & \leq C^{N} \prod_{i=1}^{r} \frac{\left\|T f^{N\left(q_{i}\right)} \mid E^{s}\left(q_{i}\right)\right\|}{\left\|T f^{N\left(q_{i}\right)} \mid E^{u}\left(q_{i}\right)\right\|} \\
& \leq C^{N} \lambda^{r} \leq C^{N} \lambda^{m}<1 / 2 .
\end{aligned}
$$

(The second inequality follows from the definition of $N\left(q_{i}\right)$.) $\quad$ q.e.d.

\section{§6. Appendix}

Let $M$ be a compact manifold without boundary. Let $f: M \rightarrow M$ be a $C^{1}$ diffeomorphism. The purpose here is to prove the following.

LEMMA A. If $z \in M$ is a non-transversal homoclinic point of $f$, then $f$ can be approximated by a diffeomorphism with $z$ as a non-hyperbolic periodic point.

Remark. A similar result was proved by Newhouse [4] in a different way.

We will apply the perturbation lemmas below to the proof of Lemma A. We fix a metric $d$ on $M$ and a $C^{1}$ metric $d^{1}$ on a neighborhood of $I$ in $\operatorname{Diff}^{1}(M)$, where $I$ is the identity of $M$.

Lemma $\mathrm{B}_{1}$. There are constants $C>0, \eta>0$ depending only on $d$ and $d^{1}$ with the following property: Let $x_{1}, x_{2} \in M$. If $d\left(x_{1}, x_{2}\right)<\varepsilon \delta$ for $0<\varepsilon$ $<\eta, 0<\delta<\eta$, then we have a $(C \varepsilon)-C^{1}$ perturbation $k$ of I, i.e. $d^{1}(k, I)$ $<C \varepsilon$, such that $k\left(x_{1}\right)=x_{2}$, and if $d\left(y, x_{1}\right)>\delta, k(y)=y$.

Lemma $B_{2}$. There are constants $C>0, \eta>0$ depending only on $d^{1}$ with the following property: Let $x \in M$ and let $L_{x}: T_{x} M \longleftarrow$ be a linear mapping. Let $I_{x}$ be the identity of $T_{x} M$. If $\left\|L_{x}-I_{x}\right\|<\varepsilon$ for $0<\varepsilon<\eta$, then 
for any $\delta>0$ we have a $(C \varepsilon)-C^{1}$ perturbation $k$ of $I$ such that $k(x)=x$, $T_{x} k=L_{x}$, and if $d(y, x)>\delta, k(y)=y$.

These facts are well-known and can be proved easily, so we omit their proofs.

Proof of Lemma A. It is sufficient to consider the case where $z \in$ $W^{s}(p) \cap W^{u}(p)$ for some fixed point $p$ because the other cases can be treated similarly. For convenience we denote by $s, u$ the dimension of $W^{s}(p)$ and $W^{u}(p)$ respectively. In what follows, $D^{s}$ (resp. $D^{u}$ ) denotes the unit disc of $\boldsymbol{R}^{s}$ (resp. $\boldsymbol{R}^{u}$ ) centered at 0 , and $B_{r}(x)$ the ball neighborhood of $x$ of radius $r>0$ in $M$.

We take a coordinate neighborhood $(U, \psi)$ of $p$ with the following properties $(1) \sim(4)$.

(1) $\psi(U)=D^{s} \times D^{u}$.

From now on, we identify $U$ with $D^{s} \times D^{u}$.

(2) $D^{s} \times\{0\} \subset W^{s}(p),\{0\} \times D^{u} \subset W^{u}(p)$.

(3) $\exists 0<\lambda<1$;

$$
\begin{array}{ll}
\left\|T_{p} f(v, 0)\right\| \leq \lambda\|v\|, & \forall v \in \boldsymbol{R}^{s}, \\
\left\|T_{p} f^{-1}(0, w)\right\| \leq \lambda\|w\|, & \forall w \in R^{u} .
\end{array}
$$

(Note $T_{p} U \approx \boldsymbol{R}^{s} \times \boldsymbol{R}^{u}$. $\|\cdot\|$ means the Euclidean norm.)

(4) $\forall x \in U \cap f(U) \cap f^{-1}(U)$;

$$
\left\|T_{x} f-T_{p} f\right\|<\alpha, \quad\left\|T_{x} f^{-1}-T_{p} f^{-1}\right\|<\alpha,
$$

where $\alpha=(1-\lambda) / 4$.

Remark. As regards (3), refer to Nitecki [5], pp. $71 \sim 73$.

Let $x \in U \cap f(U) \cap f^{-1}(U)$. For $(v, w) \in \boldsymbol{R}^{s} \times \boldsymbol{R}^{u}$, we write $\left(v_{1}, w_{1}\right)=$ $T_{x} f(v, w),\left(v_{2}, w_{2}\right)=T_{x} f^{-1}(v, w)$. Then we have (5) $\sim$ (8) below.

(5) If $\|v\| /\|w\| \leq 1 / 2,\left\|v_{1}\right\| /\left\|w_{1}\right\| \leq 1 / 2$.

Proof. Let $\pi_{1}: \boldsymbol{R}^{s} \times \boldsymbol{R}^{u} \rightarrow \boldsymbol{R}^{s}, \pi_{2}: \boldsymbol{R}^{s} \times \boldsymbol{R}^{u} \rightarrow \boldsymbol{R}^{u}$ be projections.

$$
\begin{aligned}
v_{1} & =\pi_{1} T_{x} f(v, w) \\
& =\pi_{1}\left(T_{x} f-T_{p} f\right)(v, w)+\pi_{1} T_{p} f(v, 0)+\pi_{1} T_{p} f(0, w) .
\end{aligned}
$$

Hence we have

$$
\left\|v_{1}\right\|=\alpha(\|v\|+\|w\|)+\lambda\|v\| \leq(\lambda / 2+\alpha / 2+\alpha)\|w\| \leq\|w\| / 2 .
$$

Similarly 


$$
\begin{aligned}
w_{1} & =\pi_{2} T_{x} f(v, w) \\
& =\pi_{2}\left(T_{x} f-T_{p} f\right)(v, w)+\pi_{2} T_{p} f(v, 0)+\pi_{2} T_{p} f(0, w) .
\end{aligned}
$$

and hence

$$
\left\|w_{1}\right\| \geq \lambda^{-1}\|w\|-\alpha(\|v\|+\|w\|) \geq\left(\lambda^{-1}-\alpha / 2-\alpha\right)\|w\| \geq\|w\| .
$$

Thus $\left\|v_{1}\right\| /\left\|w_{1}\right\| \leq 1 / 2$ follows.

q.e.d.

(6) If $\|w\| /\|v\| \leq 1 / 2,\left\|w_{2}\right\| /\left\|v_{2}\right\| \leq 1 / 2$.

The proof is similar to (5).

(7) If $\|w\| /\|v\| \leq 1 / 2,\left\|v_{1}\right\| \leq \lambda_{1}\|v\|$ where $\lambda_{1}=(1+\lambda) / 2$.

Proof. Decompose $v_{1}$ as in (5). Then we estimate

$$
\left\|v_{1}\right\| \leq \alpha(\|v\|+\|w\|)+\lambda\|v\| \leq(\lambda+2 \alpha)\|v\| \leq \lambda_{1}\|v\| .
$$

Thus we have (7).

q.e.d.

(8) If $\|v\| /\|w\| \leq 1 / 2,\left\|w_{2}\right\| \leq \lambda_{1}\|w\|$.

The proof is similar to (7).

We choose integers $n_{1}, n_{2}$ such that $f^{n_{1}}(z) \in D^{s} \times\{0\}, f^{-n_{2}}(z) \in\{0\} \times D^{u}$ respectively. Remark that these sets really imply their inverse images by $\psi$. Take $\delta>0$ so small that

(9) $f^{n}(z) \notin B_{\delta}\left(z_{1}\right) \cup B_{\delta}\left(z_{2}\right), \forall n ;-n_{2}<n<n_{1}$

where $z_{1}=f^{n_{1}}(z), z_{2}=f^{-n_{2}}(z)$.

Regarding $U \approx D^{s} \times D^{u}$, we write

(10) $z_{1}=\left(a_{1}, 0\right), z_{2}=\left(0, a_{2}\right)$.

Let $\varepsilon>0$ be arbitrary. We define

$$
\begin{aligned}
& F^{u}=\left\{\left(a_{1}, w\right) \in D^{s} \times D^{u} ;\|w\|<\varepsilon \delta\right\}, \\
& F^{s}=\left\{\left(v, a_{2}\right) \in D^{s} \times D^{u} ;\|v\|<\varepsilon \delta\right\} .
\end{aligned}
$$

If $n_{3}$ is sufficiently large, then $f^{-n_{3}}\left(F^{s}\right), f^{n_{3}}\left(F^{u}\right)$ are represented by $C^{1}$ mappings $h_{1}: D^{s} \rightarrow D^{u}$, and $h_{2}: D^{u} \rightarrow D^{s}$ respectively. Furthermore, we can assume

(11) $\left\|h_{1}\right\|<\varepsilon \delta,\left\|h_{2}\right\|<\varepsilon \delta$

(12) $\left\|T h_{1}\right\|<\varepsilon / 2,\left\|T h_{2}\right\|<\varepsilon / 2$.

Let $V$ be a nonzero vector in $T_{z} W^{s}(p) \cap T_{z} W^{u}(p)$. We put

(13) $V_{1}=T_{z} f^{n_{1}}(V), V_{2}=T_{z} f^{-n_{2}}(V)$.

Clearly $V_{1}$ has the form $\left(v_{1}, 0\right)$ with $v_{1} \in \boldsymbol{R}^{s}$, and $V_{2}$ has the form $\left(0, w_{2}\right)$ with $w_{2} \in \boldsymbol{R}^{u}$. We put

(14) $x_{1}=\left(a_{1}, h_{1}\left(a_{1}\right)\right), x_{2}=\left(h_{2}\left(a_{2}\right), a_{2}\right)$.

Since $x_{1}=f^{-n_{s}}\left(F^{s}\right) \cap F^{u}, x_{2}=F^{s} \cap f^{n_{s}}\left(F^{u}\right)$, it follows that 
(15) $x_{2}=f^{n_{3}}\left(x_{1}\right)$.

We put

(16) $w_{1}^{\prime}=T_{a_{1}} h_{1}\left(v_{1}\right)$.

By the definition of $h_{1}$ there is $v_{2} \in \boldsymbol{R}^{s}$ such

(17) $\left(v_{1}, w_{1}^{\prime}\right)=T_{x_{2}} f^{-n_{3}}\left(v_{2}, 0\right)$.

Let us write $\left(v_{i}^{*}, w_{i}^{*}\right)=T_{x_{2}} f^{-i}\left(v_{2}, 0\right), i=0,1, \cdots, n_{3}$. Since $\left\|w_{0}^{*}\right\| /\left\|v_{0}^{*}\right\|=0$ $<1 / 2$, it follows inductively by (6) that $\left\|w_{i}^{*}\right\| /\left\|v_{i}^{*}\right\| \leq 1 / 2, i=0,1, \cdots, n_{3}$. Hence it follows by (7) that

(18) $\left\|v_{2}\right\| \leq \lambda_{1}^{n_{3}}\left\|v_{1}\right\|$.

Likewise we put

(19) $v_{2}^{\prime}=T_{a_{2}} h_{2}\left(w_{2}\right)$.

By the definition of $h_{2}$ there is $w_{1} \in \boldsymbol{R}^{u}$ such that

(20) $\quad\left(v_{2}^{\prime}, w_{2}\right)=T_{x_{1}} f^{n_{3}}\left(0, w_{1}\right)$.

Applying (5) and (8) as above, we have

(21) $\left\|w_{1}\right\|<\lambda_{1}^{n_{3}}\left\|w_{2}\right\|$.

By (18), (21), for sufficiently large $n_{3}$ we have

(22) $\left\|w_{1}\right\| /\left\|v_{1}\right\|<\varepsilon / 2$

(23) $\left\|v_{2}\right\| /\left\|w_{2}\right\|<\varepsilon / 2$.

We define

(24) $V_{1}^{\prime}=\left(v_{1}, w_{1}+w_{1}^{\prime}\right), V_{2}^{\prime}=\left(v_{2}+v_{2}^{\prime}, w_{2}\right)$.

Then we have

$$
\begin{aligned}
T_{x_{1}} f^{n_{3}}\left(V_{1}^{\prime}\right) & =T_{x_{1}} f^{n_{s}}\left(v_{1}, w_{1}+w_{1}^{\prime}\right) \\
& =T_{x_{1}} f^{n_{3}}\left(v_{1}, w_{1}^{\prime}\right)+T_{x_{1}} f^{n_{3}}\left(0, w_{1}\right) \\
& =\left(v_{2}, 0\right)+\left(v_{2}^{\prime}, w\right) \quad(\text { by }(17),(20)) \\
& =V_{2}^{\prime} .
\end{aligned}
$$

That is,

(25) $T_{x_{1}} f^{n_{3}}\left(V_{1}^{\prime}\right)=V_{2}^{\prime}$.

By (24), (13), (22), (23) and (12), we estimate

(26) $\left\|V_{1}-V_{1}^{\prime}\right\| /\left\|V_{1}\right\|<\varepsilon$,

(27) $\left\|V_{2}-V_{2}^{\prime}\right\| /\left\|V_{2}^{\prime}\right\|<\varepsilon$.

By (26) we have a linear mapping $L_{1}: \boldsymbol{R}^{m} \rightarrow \boldsymbol{R}^{m}(m=\operatorname{dim} M)$ such that

(28) $L_{1}\left(V_{1}\right)=V_{1}^{\prime}$,

(29) $\left\|L_{1}-I\right\|<\varepsilon$, where $I$ is the identity of $\boldsymbol{R}^{m}$.

For example, take an orthogonal basis $\left\{V_{1}, e_{2}, \cdots, e_{m}\right\}$, and define $L_{1}$ by

$$
\begin{aligned}
L_{1}\left(t_{1} V_{1}+t_{2} e_{2}+\cdots+t_{m} e_{m}\right)=t_{1} V_{1}^{\prime}+t_{2} e_{2}+ & \cdots+t_{m} e_{m}, \\
& \forall t_{i} \in R ; 1 \leq i \leq m .
\end{aligned}
$$


Similarly, by (27) we have a linear mapping $L_{2}: \boldsymbol{R}^{m} \rightarrow \boldsymbol{R}^{m}$ such that

(30) $L_{2}\left(V_{2}^{\prime}\right)=V_{2}$,

(31) $\left\|L_{2}-I\right\|<\varepsilon$.

By the way, we defined $z_{1}=f^{n_{1}}(z), z_{2}=f^{-n_{2}}(z)$. By (10), (11) and (14) we have

(32) $\left\|z_{1}-x_{1}\right\|<\varepsilon \delta$,

(33) $\left\|z_{2}-x_{2}\right\|<\varepsilon \delta$.

By (29), (31), (32) and (33) we can apply Lemmas $B_{1}$ and $B_{2}$ to constructing $k \in \operatorname{Diff}^{1}(M)$ such that

(34) $k\left(z_{1}\right)=x_{1}, T_{z_{1}} k=L_{1}$.

(35) $k\left(x_{2}\right)=z_{2}, T_{x_{2}} k=L_{2}$.

(36) $k(x)=x, \forall x \notin B_{\delta}\left(z_{1}\right) \cup B_{\delta}\left(z_{2}\right)$.

(37) $k$ is a $(C \varepsilon)-C^{1}$ perturbation of the identity of $M$ ( $C$ is the one in Lemmas $\mathrm{B}_{1}$ and $\mathrm{B}_{2}$ ).

Now we shall conclude the proof. Define $g=k \cdot f \in \operatorname{Diff}^{1}(M)$. First, it follows easily from (9), (15), (34), (35) and (36) that $z$ is a periodic point of $g$ of period $n_{1}+n_{2}+n_{3}$. We show that

$$
T_{z} g^{n_{1}+n_{2}+n_{3}}(V)=V \text {, }
$$

which implies that $z$ is not hyperbolic.

$$
\begin{array}{rlrl}
T_{z} g^{n_{1}+n_{2}+n_{3}}(V) & =T_{x_{1}} g^{n_{2}+n_{3}} T_{z_{1}} k T_{z} f^{n_{1}}(V) & \quad \text { (by (9), (36)) } \\
& =T_{x_{1}} g^{n_{2}+n_{3}} L_{1}\left(V_{1}\right) & & (\text { by }(13),(34)) \\
& =T_{x_{1}} g^{n_{2}+n_{3}}\left(V_{1}^{\prime}\right) & & (\text { by }(28)) \\
& =T_{z_{2}} g^{n_{2}} L_{2}\left(V_{2}^{\prime}\right) & & (\text { by }(25),(35)) \\
& =T_{z_{2}} f^{n_{2}}\left(V_{2}\right) & & \text { (by (9), (36); (30)) } \\
& =V & & \text { (by (13)). }
\end{array}
$$

Clearly $g$ is near $f$ in $\operatorname{Diff}^{1}(M)$ by virtue of (37).

q.e.d.

\section{REFERENCES}

[1] M. Hirsch, C. Pugh and M. Shub, Invariant manifolds, Lecture Notes in Math., 583, Springer-Verlag, 1977.

[2] R. Mañé, Expansive diffeomorphisms, Proc. Symp. on Dynamical Systems-Warwick, Lecture Notes in Math., 468, 162-174, Springer-Verlag, 1975.

[ 3 ] — Contributions to the stability conjecture, Topology, 17 (1978), 383-396.

[4] S. Newhouse, Quasi-elliptic periodic points in conservative dynamical systems, Amer. J. Math., 99 (1977), 1061-1087.

[ 5 ] Z. Nitecki, Differentiable dynamics, The M.I.T. Press, Cambridge, Mass., 1971.

[6] V. Pliss, The coarseness of a sequence of linear systems of second-order differential 
equations with periodic coefficients, Diff. Uravneniya, 7 (1971), 261-270.

[ 7 ] C. Pugh, An improved closing lemma and a general density theorem, Amer. J. Math., 89 (1967), 1010-1021.

[ 8 ] S. Smale, Differentiable dynamical systems, Bull. Amer. Math. Soc., 73 (1967), $747-817$.

[ 9 ] J. Franks, Anosov diffeomorphisms, Proc. Symp. Pure Math., 14, Amer. Math. Soc. (1970), 61-93.

Kyoto University 